\title{
Physical optics modeling of 2D dielectric lenses
}

\author{
Vladimir B. Yurchenko ${ }^{1, *}$ and Ayhan Altintas ${ }^{2}$ \\ ${ }^{1}$ Institute of Radiophysics and Electronics, National Academy of Sciences of Ukraine, 12 Proskura St., \\ 61085 Kharkov, Ukraine \\ ${ }^{2}$ Electrical and Electronics Engineering Department, Bilkent University, 06800 Bilkent, Ankara, Turkey \\ *Corresponding author: v.yurchenko@nuim.ie
}

Received August 20, 2008; revised December 8, 2008; accepted December 8, 2008; posted December 11, 2008 (Doc. ID 100392); published January 27, 2009

We propose an advanced physical optics formulation for the accurate modeling of dielectric lenses used in quasi-optical systems of millimeter, submillimeter, and infrared wave applications. For comparison, we obtain an exact full-wave solution of a two-dimensional lens problem and use it as a benchmark for testing and validation of asymptotic models being considered. (C) 2009 Optical Society of America

OCIS codes: $080.3630,110.6795,220.3630,260.1960$.

\section{INTRODUCTION}

An accurate design of large-scale quasi-optical systems with dielectric lenses requires advanced wavelike asymptotic modeling of refractive components. An example of such a system is QUaD, a submillimeter-wave telescope for cosmic microwave polarization measurements [1]. For this high precision instrument to be realized, extremely accurate modeling of aberrations is needed with further minimization of their effects. The problem is particularly challenging when polarization in the focal domain has to be computed [2]. In this case, with no exact solution available due to the size limit, there is a need of advanced asymptotic methods for the wavelike modeling of lenses similar to the physical optics simulations of large reflectors [3].

Other applications for the wavelike asymptotic modeling of lenses may include optics for infrared and terahertz imaging (e.g., in security checks), automotive radars for collision-avoidance systems (where, specifically, cylindrical lenses are being used [4]), special microlenses for optical recording devices [5-8], etc.

When developing asymptotic methods, comparisons with exact solutions are of importance. In the meantime, an exact solution for electromagnetic scattering by a dielectric body is a complicated problem when the body is of a large electrical size. There are various approaches to the problem ranging from the finite difference, finite elements, and similar methods [9] to sophisticated formulations based on the integral equations [10,11] combined with regularization techniques [12-14]. Exact methods are usually limited to systems of small size, often less than ten wavelengths in diameter even in those cases when advanced solvers are available (e.g., ANSOFT HFSS software). Besides, there is only a limited number of exact solutions for dielectric lenses described in the literature.

One example of a full-wave solution is the modeling of a microcylindrical axilens [6] by using the integral equations [11] with no regularization. A rigorous regularization method $[13,14]$ is proposed for the body of special geometry considered as a resonator. A promising hybrid technique is developed for diffractive microlenses [5], where the finite difference method solves the problem in the near field and the partial plane wave (PW) propagation is used to transform the solution to the far field.

However, there appears to be no exact solutions published for conventional lens geometries, e.g., for a common biconvex lens of a standard shape. Similarly, there are no wavelike asymptotic methods developed for lenses that outperform simulations based on the ray tracing approach [2]. Conventional wave theory of lenses [2] is, in fact, the paraxial Fourier-optics, where nonparaxial cases are classified as a standing problem. The same paraxial limitations and related approximations (scalar waves, etc.) lay in the basis of quasioptical simulation models [1] that, therefore, do not account for polarization effects and nonparaxial issues in the interaction of the vector electromagnetic waves with the dielectric lens body.

In the meantime, lenses of intermediate (quasi-optical) size (tens to a few hundreds of radiation wavelength) do, usually, require both the large-angle incidence and the vector character of the electromagnetic field to be accounted for while being too large for exact simulations. It is this kind of lens enhanced asymptotic modeling that is required for emerging practical applications.

The aim of this paper is to present enhanced asymptotic simulation methods for dielectric lenses of the quasioptical range and to provide validation for these methods by comparing them with full-wave analytic solutions obtained for two-dimensional (2D) focusing lenses of a conventional biconvex profile.

In Section 2, we obtain a full-wave analytic solution to the problem of an electromagnetic wave being focused by a 2D dielectric lens with a typical cross section profile formed by two circular arcs as shown in Fig. 1. In this solution, we consider the cases of $\mathrm{PW}$ and cylindrical wave (CW) incidence on the lens surface and compute the wave fields behind the lens for two orthogonal polarizations, TE and TM, respectively. Then, in Section 3, we describe asymptotic methods appropriate for quasi-optical lenses that utilize the physical optics approach. Finally, we com- 


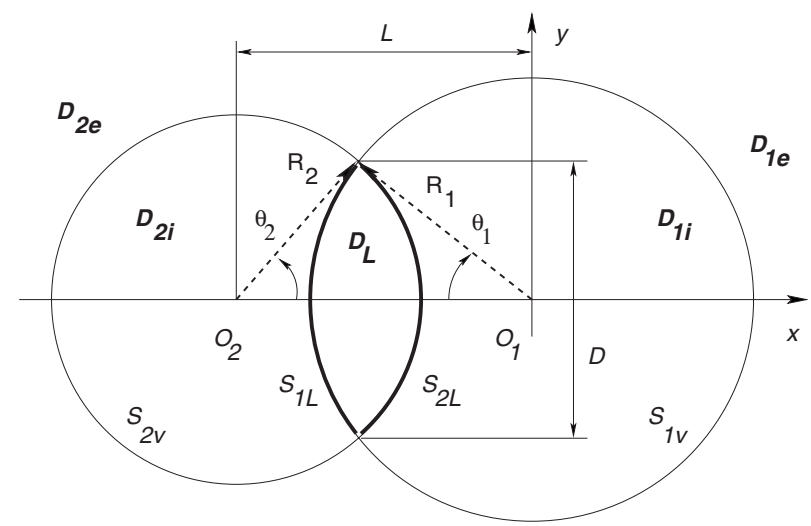

Fig. 1. Geometry of a 2D convex dielectric lens (cross section profile). The excitation wave (PW or CW) of either TM $(\mathbf{E} \| \hat{\mathbf{z}})$ or TE $(\mathbf{H} \| \hat{\mathbf{z}})$ polarizations is incident from the left.

pare simulation results produced by asymptotic methods with exact solutions at various values of lens parameters.

\section{FULL-WAVE SIMULATION OF A TWO-DIMENSIONAL FOCUSING DIELECTRIC LENS}

\section{A. Simulation Method}

Full-wave analytic solutions for dielectric lenses in the 2D problems shown in Fig. 1 can be obtained by direct methods when using CW expansions of incident and scattered fields with respect to $O_{1}$ and $O_{2}$ frames associated with circular arcs $S_{1 L}$ and $S_{2 L}$ that form the lens cross section profile.

The lens geometry is defined by the lens diameter $D$ and the arc curvature radii $R_{1,2}$ so that the lens angular half-size $\theta_{1,2}$ as viewed from the origin $O_{1,2}$ is $\theta_{1,2}$ $=\arcsin \left(D / 2 R_{1,2}\right)$, the distance between the origins is $L$ $=R_{1} \cos \left(\theta_{1}\right)+R_{2} \cos \left(\theta_{2}\right)$, and the lens thickness along the $x$-axis is $t=R_{1}+R_{2}-L$.

In this paper, we consider the waves of $\mathrm{TE}(\mathbf{H} \| \hat{\mathbf{z}})$ and TM $(\mathbf{E} \| \hat{\mathbf{z}})$ polarization with two kinds of wave sources, namely, the uniform PW and CW incident on the lens from the left (Fig. 1). The incident wave in the PW case propagates along the positive direction of the $x$-axis. In the $\mathrm{CW}$ case, the incident wave is radiated from a line source located at a certain distance in front of the lens. In the $x y$ plane, it is an omnidirectional source placed at the $x_{C}, y_{C}$ point on the left side of the lens with $x_{C}<-R_{1}$ and $y_{C}=0$ being the source coordinates in the $O_{1}$ frame (the left and right apex points of the lens have coordinates $x_{L}=-R_{1}$ and $x_{R}=-R_{1}+t$, respectively).

Using the $O_{1}$ frame, we expand the relevant $z$-component of the field $\left(U=E_{z}\right.$ in the TM cases and $U$ $=H_{z}$ in the TE cases) in cylindrical functions in the domains $D_{1 e}, D_{1 i}$, and $D_{L}$ as follows:

$$
\begin{gathered}
U=\sum_{n}\left[A_{n} J_{n}\left(k r_{1}\right)+B_{n} Y_{n}\left(k r_{1}\right)\right] e^{i n \phi_{1}}, \\
\left(r_{1}, \phi_{1}\right) \in D_{L}
\end{gathered}
$$

$$
\begin{gathered}
U=\sum_{n} C_{n} J_{n}\left(k r_{1}\right) e^{i n \phi_{1},} \\
\left(r_{1}, \phi_{1}\right) \in D_{1 i}, \\
U=\sum_{n}\left[F_{n} H_{n}\left(k r_{1}\right)+b_{n}\left(k r_{1}\right)\right] e^{i n \phi_{1}}, \\
\left(r_{1}, \phi_{1}\right) \in D_{1 e},
\end{gathered}
$$

where $A_{n}, B_{n}, C_{n}$, and $F_{n}$ are the unknown expansion coefficients, $J_{n}(\cdot), Y_{n}(\cdot)$ and $H_{n}(\cdot)=H_{n}^{(2)}(\cdot)$ are the Bessel, Neumann, and Hankel functions of order $n$ (the time dependence of the wave field is assumed to be $\exp (i \omega t)), k$ $=2 \pi / \lambda$ and $k_{d}=k \sqrt{\varepsilon}$ are the wavenumbers in free space and in the lens dielectric material of (complex) relative permittivity $\varepsilon$, respectively, $\lambda$ is the free-space wavelength, $r_{1}$ and $\phi_{1}$ are the radial and angular coordinates in the $O_{1}$ frame, $R_{1}$ and $R_{2}$ are the curvature radii of the entrance $\left(S_{1 L}\right)$ and exit $\left(S_{2 L}\right)$ lens surfaces, and the expansion domains are defined as $D_{1 e, 2 e}=\left\{r_{1,2}: r_{1,2}>R_{1,2}\right\}$, $D_{1 i, 2 i}=D_{1,2}-D_{L}, D_{1,2}=\left\{r_{1,2}: r_{1,2}<R_{1,2}\right\}$, and $D_{L}=D_{1} \cap D_{2}$.

The expansions satisfy the Helmholtz equations in the relevant domains and boundary conditions of (a) no singularity at the origin (there is neither source nor sink at point $O_{1}$ ) and (b) no incoming waves from infinity except the given source wave (the radiation boundary condition). The terms $b_{n}$ represent the source wave and take on the form $b_{n}\left(k r_{1}\right)=i^{-n} J_{n}\left(k r_{1}\right)$ in the PW case and $b_{n}\left(k r_{1}\right)$ $=(-1)^{n} J_{n}\left(k r_{1}\right) H_{n}\left(k L_{C}\right)$ in the $\mathrm{CW}$ case, where $L_{C}=\left|C O_{1}\right|$ is the distance from the source point $C$ to the frame origin $O_{1}$ (the source distance from the lens front surface is $\left.L_{\mathrm{CL}}=L_{C}-R_{1}\right)$.

The Neumann addition theorem for cylindrical functions is used for transforming the expansions from one frame $\left(\mathrm{O}_{1}\right)$ to another $\left(\mathrm{O}_{2}\right)$ when imposing boundary conditions for tangential field components at the lens surfaces $S_{1 L}$ and $S_{2 L}$. Then, by using the orthogonality of angular harmonics, we arrive at the set of linear algebraic equations with respect to expansion coefficients as follows:

$$
\begin{gathered}
\sum_{n, m}\left\{\left[A_{n} J_{m-n}\left(k_{d} L\right)+B_{n} Y_{m-n}\left(k_{d} L\right)\right] J_{m}\left(k_{d} R_{2}\right)\right. \\
\left.-C_{n} J_{m-n}(k L) J_{m}\left(k R_{2}\right)\right\} S_{\mathrm{mp}}\left(\theta_{2}\right)=0 \\
\sum_{n, m}\left\{\eta\left[A_{n} J_{m-n}\left(k_{d} L\right)+B_{n} Y_{m-n}\left(k_{d} L\right)\right] J_{m}^{\prime}\left(k_{d} R_{2}\right)\right. \\
\left.-C_{n} J_{m-n}(k L) J_{m}^{\prime}\left(k R_{2}\right)\right\} S_{\mathrm{mp}}\left(\theta_{2}\right)=0 \\
\sum_{n}\left\{\left[A_{n} J_{n}\left(k_{d} R_{1}\right)+B_{n} Y_{n}\left(k_{d} R_{1}\right)\right] P_{\mathrm{np}}(\tau)+C_{n} J_{n}\left(k R_{1}\right) S_{\mathrm{np}}(\tau)\right\} \\
-F_{p} H_{p}\left(k R_{1}\right)=b_{p}\left(k R_{1}\right) \\
\sum_{n}\left\{\eta\left[A_{n} J_{n}^{\prime}\left(k_{d} R_{1}\right)+B_{n} Y_{n}^{\prime}\left(k_{d} R_{1}\right)\right] P_{\mathrm{np}}(\tau)+C_{n} J_{n}^{\prime}\left(k R_{1}\right) S_{\mathrm{np}}(\tau)\right\} \\
-F_{p} H_{p}^{\prime}\left(k R_{1}\right)=b_{p}^{\prime}\left(k R_{1}\right)
\end{gathered}
$$


where $p=0, \pm 1, \ldots, S_{\mathrm{mp}}(\theta)=\sin [(m-p) \theta] /[\pi(m-p)]$ if $m$ $\neq p, S_{\mathrm{mp}}(\theta)=\theta / \pi$ if $m=p, P_{\mathrm{np}}(\tau)=\delta_{\mathrm{np}}-S_{\mathrm{np}}(\tau), \tau=\pi-\theta_{1}, \eta$ $=\sqrt{\varepsilon}$ in TM and $\eta=1 / \sqrt{\varepsilon}$ in TE polarizations, and prime ( $'$ ) denotes the derivative of a function over argument. By introducing reasonably large truncation numbers $N$ and $M$ for summations in $n$ and $m$, respectively, we could find sufficiently convergent numerical solutions to Eqs. (4)-(7) that, by substitution into Eqs. (2) and (3), allowed us to compute fields in the focal domain behind the lens.

Because of rapid growth or decay of cylindrical functions with angular harmonic index $n$, direct solutions of this kind are limited to lenses of relatively small size (truncation numbers have to be limited from above). In practice, we could simulate lenses with the curvature radii $R_{1}, R_{2}$, and lens diameter $D$ up to about $12 \lambda$ when having the lens refractive index $\widetilde{n}=1.5$ (notice, the lenses used in, e.g., car radar applications [4] are of a similar size $D \approx 10 \lambda$ ). A further increase of the lens size can be achieved by implementing regularization methods.

\section{B. Simulation Results}

The results showing the wave focusing effects in TM and TE polarizations for both the $\mathrm{PW}$ and the $\mathrm{CW}$ incident

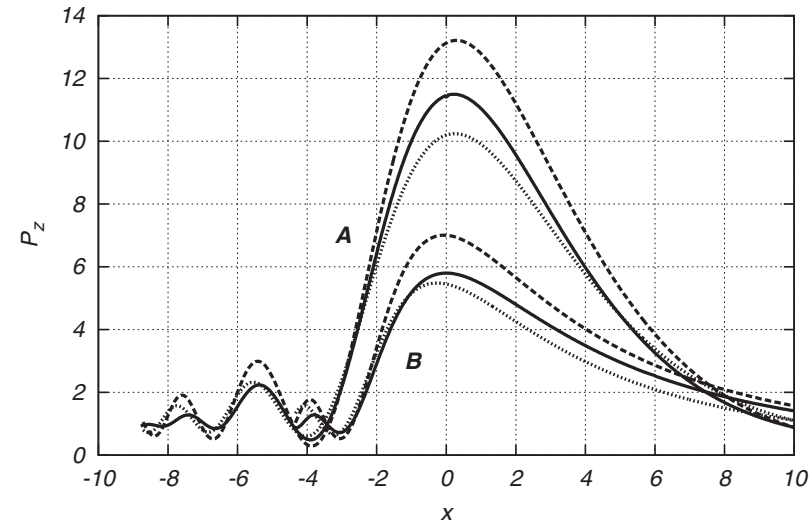

(a)

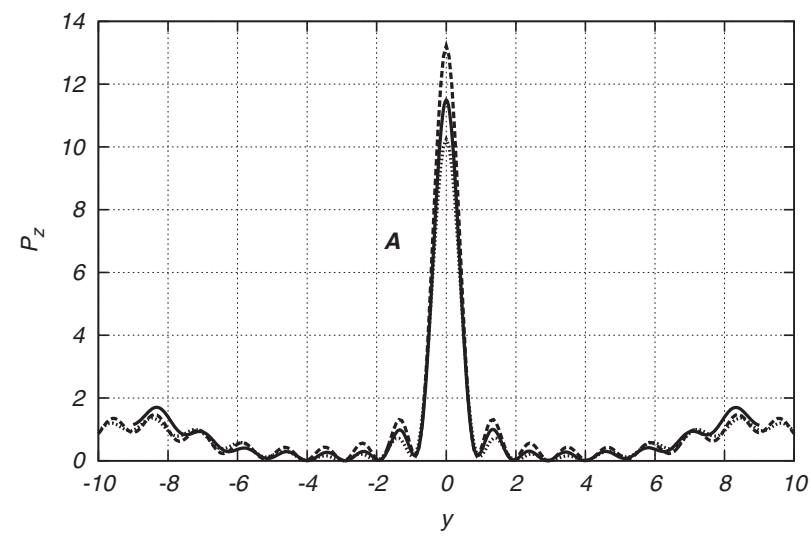

(b)

Fig. 2. Exact (solid curves) and asymptotic solutions (dashed curves for RS2 and dotted curves for KU models, see below) in the case of TM PW incidence on a symmetric 2D dielectric lens; (a) $x$ and (b) $y$ cuts of $P_{z}$ power distribution behind the lens. Here, $\tilde{n}=1.5, R_{1}=R_{2}=D, \theta_{1}=\theta_{2}=30^{\circ} ; \mathrm{A}$ and $\mathrm{B}$ groups of curves correspond to $D=12 \lambda\left(x_{R}=-8.7 \lambda\right)$ and $D=6 \lambda\left(x_{R}=-4.3 \lambda\right)$, respectively. fields are plotted in Figs. 2 and 3. We choose the lens refractive index $\tilde{n}=1.5$ so that, in the case of $\mathrm{PW}$ incidence, the geometrical focal point $F_{1}$ of a thin symmetric lens coincides with the frame origin $O_{1}$ (the case of $\mathrm{PW}$ incidence was examined in detail in our earlier work [15]).

Power density $P_{z}=\left|U_{z}\right|^{2}$ associated with $E_{z}$ and $H_{z}$ field components of TM and TE waves, respectively, is shown in the focal domain of lens in transverse $(y)$ and longitudinal $(x)$ cuts passing through the focal point $F_{1}$. A common feature of these and other simulations is that the TE polarized waves produce a greater power density at the focal point as compared to the TM waves of the same incident power. This is due to the difference in TE and TM wave transmissions at oblique incidence at the lens surfaces as expressed by the Fresnel coefficients. The effect is more complicated for small lenses and CWs [Fig. 3(a)], though, typically, it remains of a similar kind.

The second common feature is that the width of the focal spot defined as the distance between the first minima of the field is twice the wavelength in all the cases of PW focusing [Fig. 2(b)]. In the meantime, the width of diffraction fringes is one wavelength for lenses of a different size with nearly identical patterns in both polarizations. In

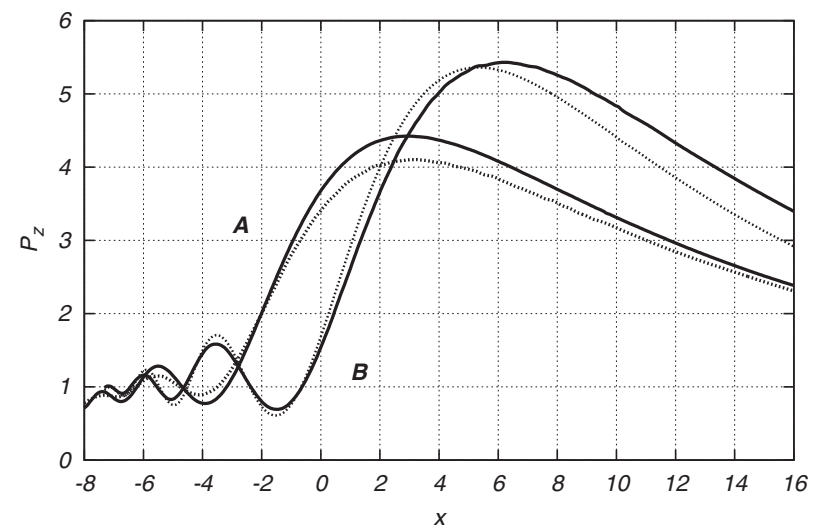

(a)

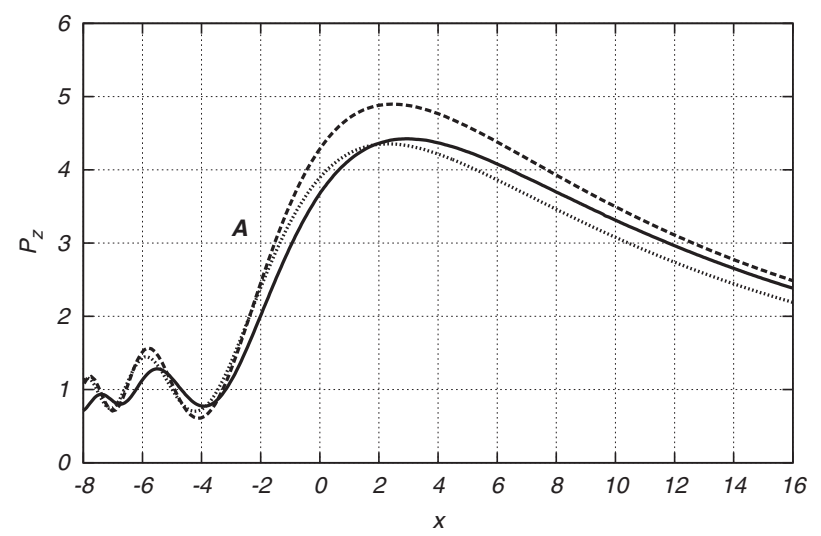

(b)

Fig. 3. (a) Exact solutions in TE (solid curves) and TM (dotted curves) cases of CW incidence on a lens with $R_{1}=10 \lambda$ when (A) $D=R_{2}=8 \lambda \quad\left(x_{R}=-8.0 \lambda\right)$ and (B) $D=R_{2}=10 \lambda\left(x_{R}=-7.2 \lambda\right)$ at $L_{C}$ $=30 \lambda\left(L_{C L}=20 \lambda, \tilde{n}=1.5\right)$ and (b) comparison of exact (solid curve) and asymptotic solutions (dashed curves for RS2 and dotted curves for KU models, see below) in case A of TE CW incidence. 
the case of $\mathrm{CW}$ focusing, the focal spot is, typically, wider and the focusing effect is more complicated (see below).

In general, there are some intrinsic resonant effects associated with lenses, which are especially pronounced in the narrowband applications. With increasing the size of the lens and the bandwidth of radiation, the resonant effects smear out and become insignificant (especially in the presence of losses).

\section{ASYMPTOTIC WAVELIKE MODELING OF DIELECTRIC LENSES}

\section{A. Two-Term Kirchhoff Approximation}

Asymptotic approximations proposed in this paper are based on various forms of diffraction integrals that represent wave propagation from the lens exit surface to the observation points behind the lens. The integral transforms of this or a similar kind are needed in any nearfield to far-field propagation in free space.

Inside the lens, propagation of the incident wave from the entrance surface $S_{1 L}$ to the exit surface $S_{2 L}$ can be evaluated by using the ray tracing through the lens body. In this paper, the ray tracing is implemented on account of (a) the transmission Fresnel coefficients at $S_{1 L}$ and $S_{2 L}$ surfaces, (b) phase increments along the rays inside the lens, and (c) wave amplitude increments (decrements) due to the ray convergence (divergence) because of refraction. This evaluation is quite accurate for typical lenses because of a relatively short ray propagation path inside the lens as compared to the lens transverse dimensions.

The first method we consider is based on the two-term Kirchhoff diffraction integral. The integral is evaluated over the extended exit surface $S_{2}$ that consists of the lens exit surface $S_{2 L}$ and the free-space surface $S_{2 F}$ extended from the lens rim to infinity in the transverse direction. The first term depends on the wave amplitude $U$ that is directly evaluated at the exit surface $S_{2}$ as explained above. The second term depends on the normal derivative of $U$ at $S_{2}$ that is approximated by using the $U$ values at $S_{2}$ and the set of ray directions that define the local inclination of the wavefront (we denote this approach as the KU model).

\section{B. One-Term Rayleigh-Sommerfeld Formulation Modified for Curved Surfaces}

Another asymptotic model is obtained by starting with a one-term Rayleigh-Sommerfeld diffraction integral and modifying it for nonplanar (curved) exit surfaces. Since the idea of the original Rayleigh-Sommerfeld formulation is the choice of the Green's function that vanishes at the plane integration surface (thus removing the second poorly defined term in the Kirchhoff formulation), we further modify the Green's function to make it identically zero at the curved lens exit surface $S_{2 L}$ while using the original form at the planar free-space surface $S_{2 F}$ outside the lens. We denote this formulation as an RS2 model.

Notice, the Green's function defined above is an exact one for the domain of a given geometry. It means that, if the field on the exit surface $S_{2}$ is known precisely, the formulation is exact. Therefore, the RS2 model is superior to other forms of the Rayleigh-Sommerfeld approach proposed for curved surfaces as, e.g., those where no modifi- cation of the Green's function is made [7] or an alternative four-term Green's function is introduced [8] that, however, generates entirely wrong results when tested in our simulations.

\section{Comparison of Exact and Asymptotic Solutions}

An important goal of this paper is the validation of asymptotic models by comparing their results one with another and with available exact solutions when considering various kinds of lenses. Therefore, we examine the performance of asymptotic models and relevant software codes in a broad domain of lens parameters.

The comparison of KU and RS2 simulations with fullwave solutions is presented in Figs. 2 and 3(b). In these cases, the lenses are characterized by the focal length $f$ $\sim D>\lambda$. In these and other simulations [15], KU and RS2 models appeared to be, in general, sufficiently accurate.

Inaccuracy in asymptotic models may arise due to (a) neglect of the edge diffraction and the internal reflection in the lens, (b) inaccuracy of the ray model for the wave propagation inside the lens, (c) approximations in the amplitude and phase evaluation of the wave transmitted through the lens exit surface, and (d) additional approximations in the KU model needed for the evaluation of a normal derivative of the wave field at the lens exit surface $S_{2 L}$.

A typical feature observed in asymptotic solutions is that the values of power density $P_{z}(x, y)$ computed with $\mathrm{KU}$ and RS2 models provide the lower and the upper bounds for the exact solution, respectively. Therefore, by combining both the KU and RS2 results, we can, generally, estimate and improve the accuracy of approximate asymptotic simulations.

Capabilities of asymptotic models in computing 2D field distributions are shown in Fig. 4. Figure 4(a) shows an exact power pattern (in relative units) in the focal domain of the lens presented in case B of Fig. 3 in TE polarization (CW excitation by the line source at $L_{C}=30 \lambda$ when $D=R_{1}=R_{2}=10 \lambda$ ). One can see a pair of specific fringes originating at the lens rim due to the edge diffraction (the waves aside the lens are partially suppressed by this effect near the lens rim). The lens is suspended freely in space as shown in Fig. 1.

Figure 4(b) shows the pattern computed for the same lens with the RS2 asymptotic model.There is a clear similarity between the patterns in Figs. 4(a) and 4(b) in all the main features, such as the focal spot, basic diffraction fringes in the focal domain, two rather bright caustics, and even a specific interference structure along the caustics consistent with fringes behind the lens. A noticeable difference is only the absence of significant edge diffraction and no suppression of waves aside the lens, respectively (there is no special account for edge diffraction in this model yet).

In practice, the lens is usually mounted into a stop that is not transparent for the incident waves. The stop is not accounted for by an exact solution described above. The asymptotic models can, however, easily account for the stop. Figure 4(c) shows the pattern computed with the RS2 model for the same lens as described above, which is mounted into the stop. The stop is simulated as an absorbing screen orthogonal to the $x$-axis with an aperture 


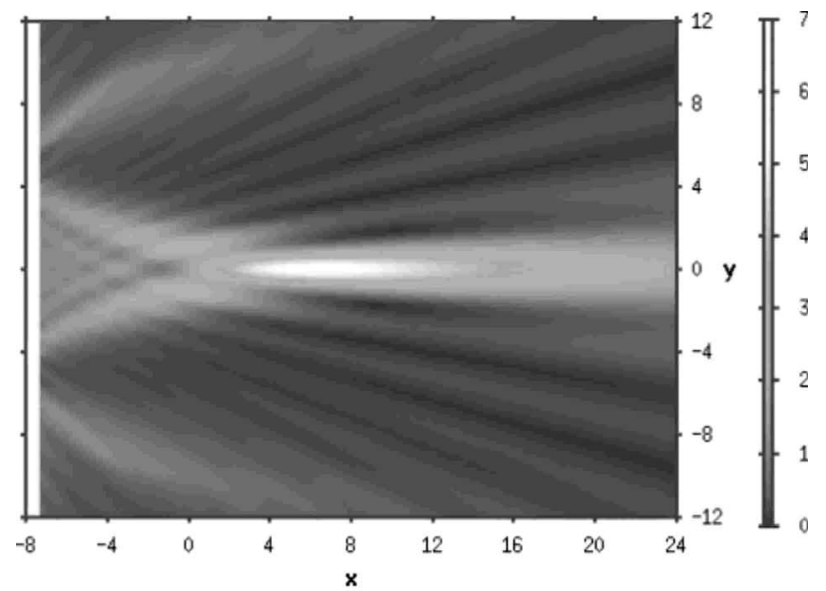

(a)

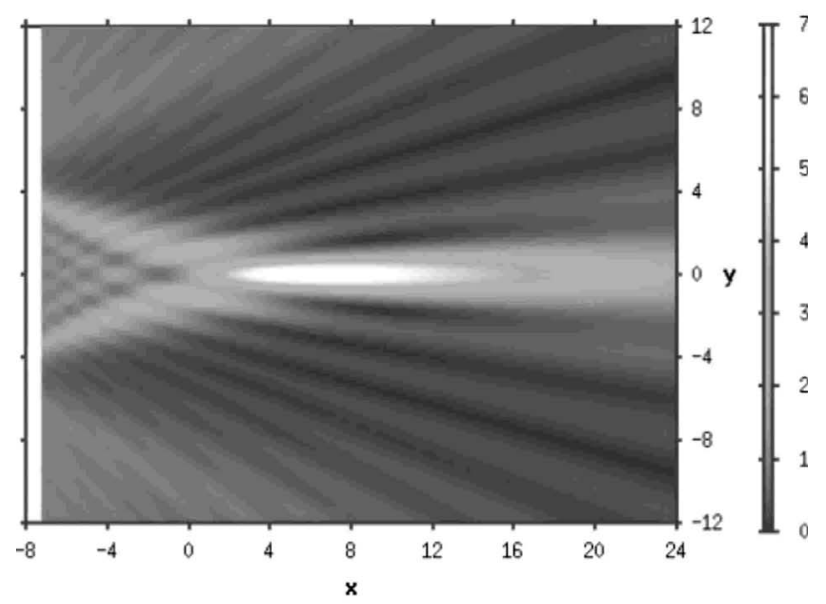

(b)

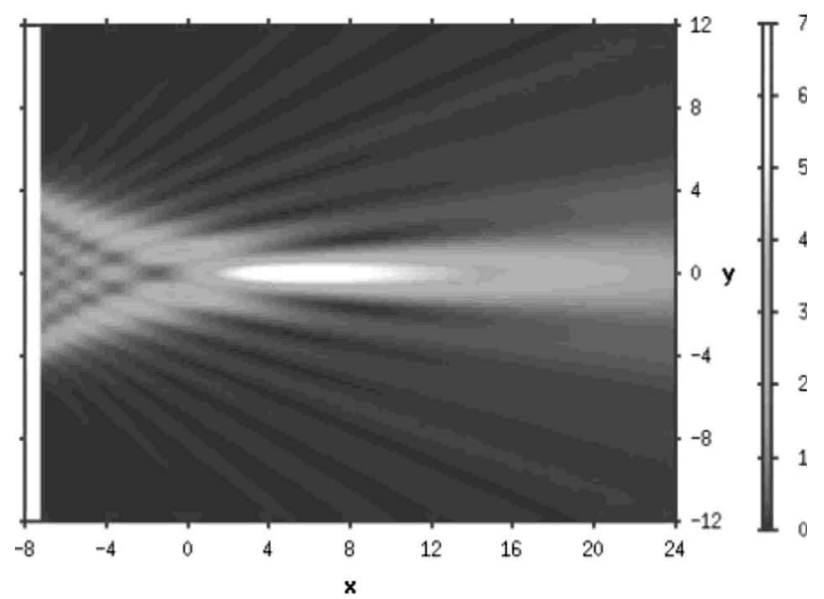

(c)

Fig. 4. Power patterns $P_{z}(x, y)$ behind the lens in case B of Fig. 3 in TE CW incidence. (a) Exact solution for a lens suspended in free space, (b) RS2 simulation for the same case, and (c) RS2 simulation for the same lens mounted into a stop.

of size $D$ where the lens is fixed by its rim. The pattern shows significant suppression of the side waves behind the screen except those diffracted by the lens, though all the wave features in the focal domain are the same as in Figs. 4(a) and 4(b).

\section{Asymptotic Solutions for Large- and Small-Scale Lenses}

Asymptotic approximations should be particularly useful for large-scale lenses, where exact solutions are not accessible while asymptotic methods remain efficient. Generally, short-wave asymptotic methods are more accurate for large scattering objects as compared to small ones. The rule is valid for dielectric lenses as well, though in this case, there are two kinds of parameters that control the accuracy, namely, (a) the lens surface curvature radii $R_{i}$ and (b) their ratio to the lens diameter (equivalently, the lens aperture angles $\theta_{i}$ ) rather than the lens diameter $D$ alone.

The discrepancy between KU and RS2 models decreases with increasing the size of the lens of the same profile. For example, the discrepancy in $P_{z}$ at the focal point of a rather bulky symmetric lens in case A of Fig. 2 (a) decreases from nearly $25 \%$ when $D=R_{1}=R 2=12 \lambda$ to $10 \%$ and $5 \%$ for a lens of the same shape at $D=R_{1}=R 2$ $=100 \lambda$ and $D=R_{1}=R 2=300 \lambda$, respectively. Yet, the discrepancy for bulky lenses such as this $(t / D=0.27)$ is relatively significant, being the consequence of general difficulty in solving large-angle $\left(\theta_{1}=\theta_{2}=30^{\circ}\right)$ nonparaxial lens problems [2].

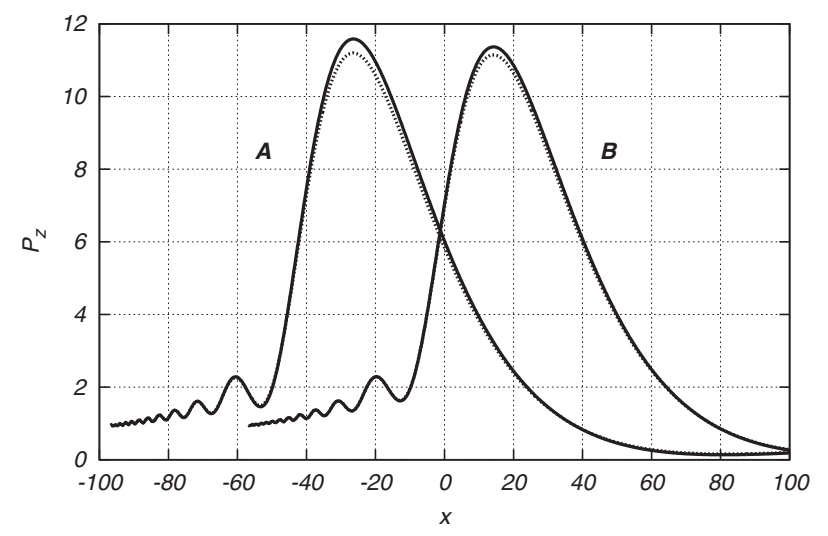

(a)

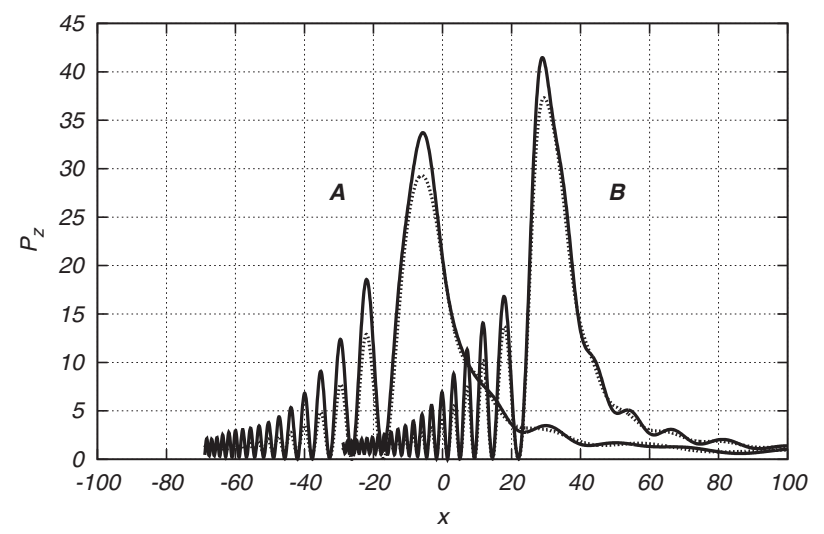

(b)

Fig. 5. Power density $P_{z}$ (TM PW case) in the focal domain of asymmetric lenses of diameter (a) $D=30 \lambda$ and (b) $D=90 \lambda(\tilde{n}$ $=1.5$ ) when either $R_{1}=100 \lambda, R_{2}=60 \lambda$ (A orientation of lens), or $R_{1}=60 \lambda, R_{2}=100 \lambda$ (B orientation) computed with KU (dotted curves) and RS2 (solid curves) asymptotic methods. 
Another example is presented in Fig. 5, which compares the effects in relatively thin and thick asymmetric lenses of a sufficiently large scale. It shows the power density $P_{z}$ (TM PW case) in the focal domain of asymmetric lenses of diameter (a) $D=30 \lambda$ and (b) $D=90 \lambda$ when either $R_{1}=100 \lambda, R_{2}=60 \lambda$ (A orientation of lens), or $R_{1}$ $=60 \lambda, R_{2}=100 \lambda$ (B orientation) computed with KU and RS2 methods (these are relatively large lenses with focal length $f>D \gg \lambda$ that cannot be easily simulated by exact methods).

Figure 5(a) illustrates a good agreement (better than $2 \%$ ) of asymptotic methods for a lens of a limited diameter $(D=30 \lambda)$ in the case of relatively large radii $R_{1,2}$ when $\theta_{1}=8.6^{\circ}, \theta_{2}=14.5^{\circ}$ (in $\mathrm{B}$ orientation) and the lens thickness is $t=3 \lambda$. In the meantime, Fig. $5(\mathrm{~b})$ shows quite a significant discrepancy between asymptotic methods (up to $10 \%)$ when the lens diameter is three times greater $(D$ $=90 \lambda)$ at the same radii $R_{1,2}$ as before. In this case, $\theta_{1}$ $=48.6^{\circ}, \theta_{2}=26.7^{\circ}$, and the lens thickness $t=31 \lambda$ (the relative lens thickness is $t / D=0.10$ and $t / D=0.34$ in Figs. 5(a) and $5(\mathrm{~b})$, respectively). Figure 5(b) also shows a certain advantage of the $\mathrm{B}$ orientation of large asymmetric lenses for obtaining a sharper focusing of incident PWs.

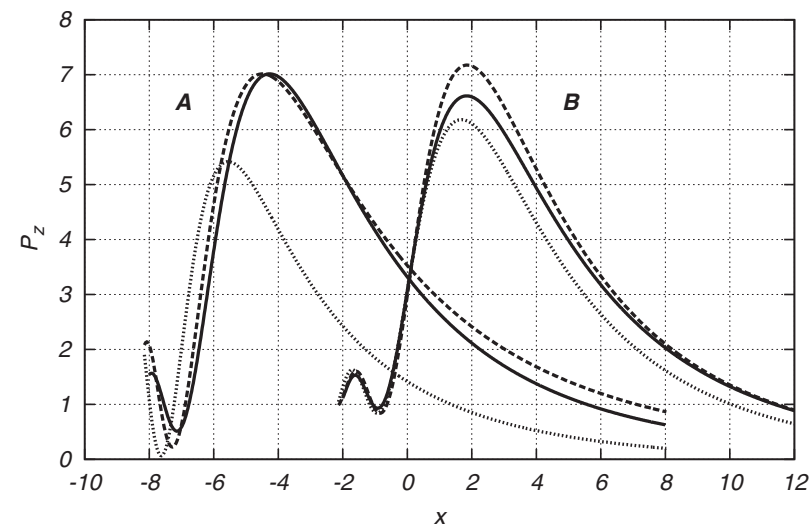

(a)

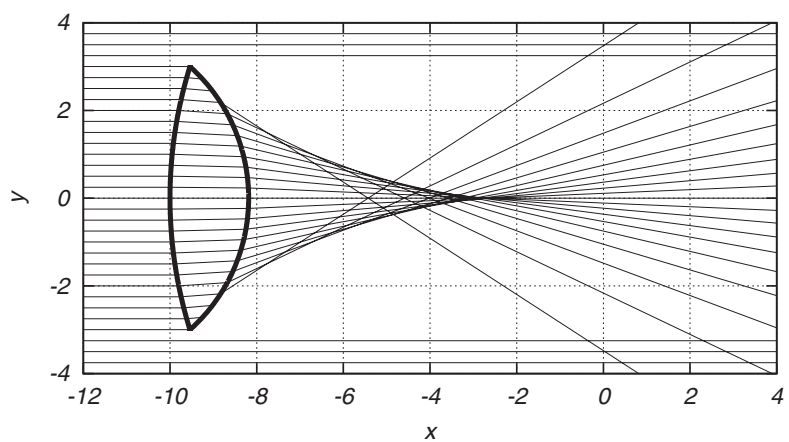

(b)

Fig. 6. (a) Power density $P_{z}$ (TE PW case) in the focal domain of an essentially asymmetric lens of small size in A and B orientations $\left(\tilde{n}=1.5, D=6 \lambda, R_{1}=10 \lambda, R_{2}=4 \lambda\right.$ in case A when $\theta_{1}$ $=17.5^{\circ}, \theta_{2}=48.6^{\circ}$ ) computed with KU (dotted curves) and RS2 (dashed curves) asymptotic methods as compared to exact solutions (solid curves), and (b) total internal reflection near the lens rim in case A as illustrated by ray tracing (a few rays near the rim in this plot do not propagate through the lens).

\section{E. Optimal Asymptotic Approximations for Asymmetric} Lenses

The results above are obtained for symmetric or slightly asymmetric lenses that have comparable surface curvature radii $R_{1} \sim R_{2}$. For asymmetric lenses with $R_{1} \gg R_{2}$ and a relatively small focal length $(f \sim D \sim 10 \lambda)$ we observe, however, certain problems. For these lenses, the results of two asymptotic methods may differ significantly [Fig. 6(a) dashed and dotted curves in case A]. The effect is less pronounced for greater lenses of a similar shape, though the tendency remains the same.

A detailed analysis revealed that, in these cases, we observe the effect of total internal reflection of rays near the lens rim. Due to this effect, no wave can propagate through the lens in the rim area [Fig. 6(b)]. Moreover, the area near the rim where the wave would experience total internal reflection may be quite significant. Notice, even though both the KU and the RS2 models take account of this effect by assigning zero amplitude to the wave in this area on the exit surface $S_{2 L}$, inconsistency of approximate field distributions (and also of a normal derivative in the $\mathrm{KU}$ model) in this domain may be much too significant for certain models to be reliable.

To compare the applicability of different models for asymmetric lenses, we computed exact solutions in those

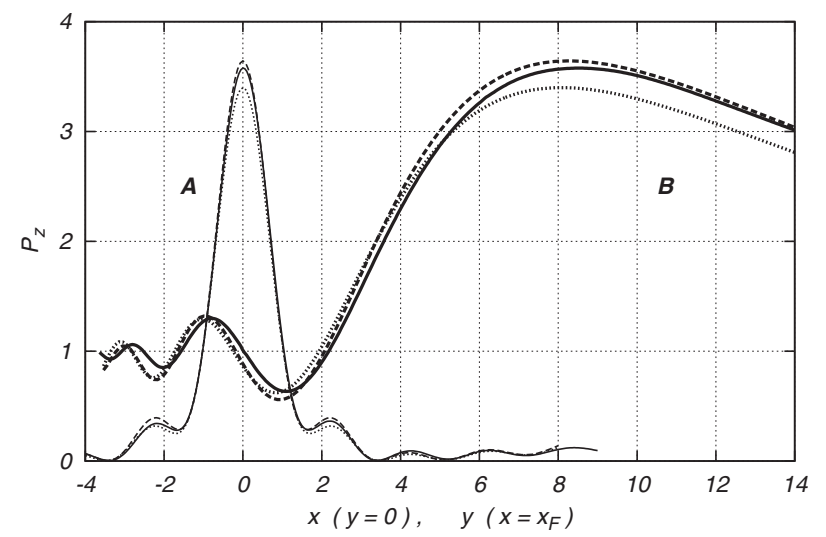

(a)

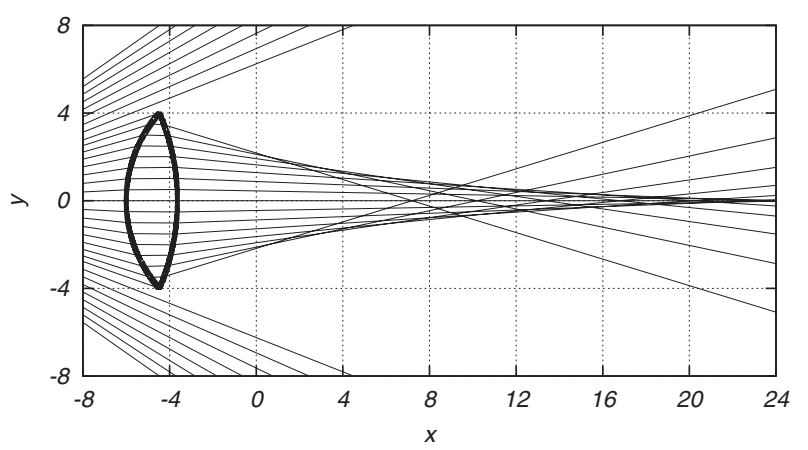

(b)

Fig. 7. (a) Power density $P_{z}(y)$ at $x=x_{F}$ (A curves) and $P_{z}(x)$ at $y=0$ (B curves) computed by KU (dotted curve) and RS2 (dashed curve) asymptotic methods in the comparison with an exact solution (solid curve), and (b) set of rays in the case of CW incidence on an asymmetric lens in the B orientation when $D=8 \lambda$, $R_{1}=6 \lambda, R_{2}=10 \lambda, L_{C}=16 \lambda$, and $\tilde{n}=1.5$ (TE polarization). 
cases where it was possible [Fig. 6(a) solid curves]. It appears that exact solutions are also quite difficult to obtain in those cases when the effect of total internal reflection is observed.

The comparison of asymptotic and exact solutions for asymmetric lenses with curvature radii $R_{1} \gg R_{2}$ has shown that the Rayleigh-Sommerfeld model RS2 based on the modified Green's function has an advantage over the Kirchhoff formulation KU. The reason for the advantage of the RS2 model over the KU one (and, indeed, over other Rayleigh-Sommerfeld models [5-8]) is that, in this form, it uses an integral representation with an exact Green's function, which is chosen to be precisely matching the lens geometry. In all the cases considered, the RS2 method proved to be capable of a rather accurate representation of waves of asymmetric lenses when compared to the exact solutions, whereas the KU method failed substantially in these circumstances.

As a final example, Fig. 7 shows the power density $P_{z}$ and a set of rays in the case of $\mathrm{CW}$ incidence on an asymmetric lens in the $\mathrm{B}$ orientation (TE polarization) when $D=8 \lambda, R_{1}=6 \lambda, R_{2}=10 \lambda$, and $L_{C}=16 \lambda$. The transverse cut of $P_{z}(x, y)$ through the focal point $F$ defined as the point of maximum power shows a wider focal spot in the case of CW focusing as compared to the PW cases (nearly $4 \lambda$ against $2 \lambda$ in the PW cases).

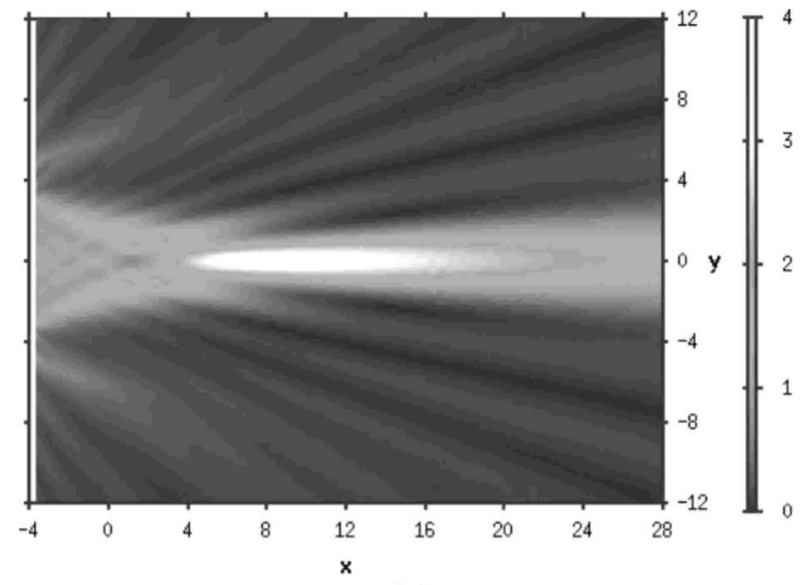

(a)

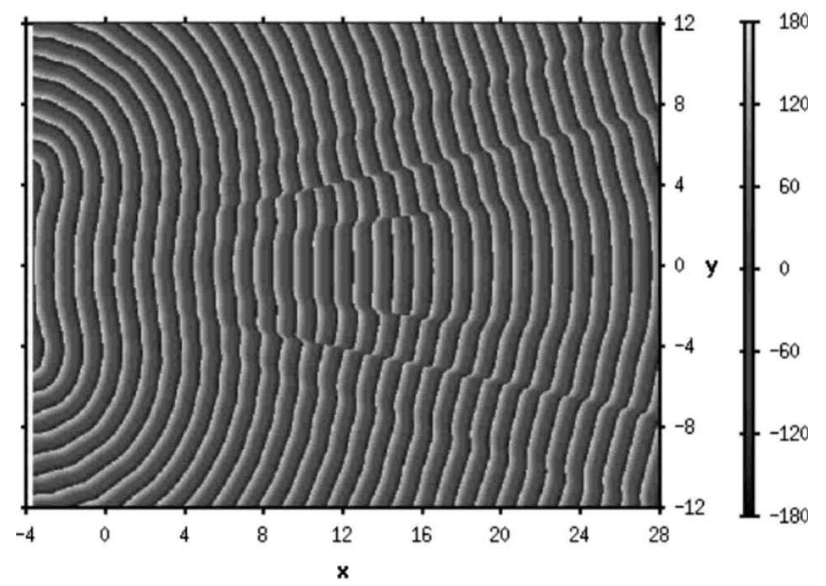

(b)

Fig. 8. (a) Power and (b) phase patterns computed by an exact method in the case of Fig. 7.
Another important feature is a significant shift of the focal point $F$ closer to the lens as compared to the (approximate) paraxial geometrical focus $F_{0}\left(x_{F}=8 \lambda\right.$ against $x_{F_{0}} \approx 24 \lambda$ ). This effect is a common feature of converging diffracted waves [16]. Notice, however, that a similar shift in the PW case shown in Fig. 6 is far less significant.

The issue is well illustrated in Fig. 8, which shows both the $2 \mathrm{D}$ power and phase patterns behind the lens. The phase pattern in Fig. 8(b) shows the phase slippage that occurs in the focal area in the process of wave focusing. The actual phase slippage in this example happens at $x_{P} \approx 14 \lambda$, i.e., about halfway between the maximum power focal point and the geometrical focus.

\section{CONCLUSIONS}

We proposed and analyzed a few asymptotic wavelike approximations for the accurate and efficient modeling of dielectric lenses used in quasi-optical systems of millimeter, submillimeter, and infrared wave applications. For comparison, we developed an exact full-wave analytic solution of a two-dimensional focusing lens problem and used it as a benchmark for testing and validation of the asymptotic models being proposed.

The main asymptotic formulations considered are the two-term Kirchhoff model with an appropriate approximation of the normal derivative of complex wave amplitude at the lens surface (KU) and the one-term RayleighSommerfeld diffraction integral formulation modified for nonplanar (curved) exit lens surfaces (RS2). The Rayleigh-Sommerfeld approximation modified for curved surfaces (RS2 model) is found to be more general and better suited for various kinds of dielectric lenses including symmetric and asymmetric, thin and thick, and rather large and relatively small lenses.

Both the Kirchhoff model (KU) and the RayleighSommerfeld representation modified for nonplanar surfaces (RS2) are remarkably accurate for large lenses $(f, D \gg \lambda)$, where no total internal reflection effects occur (i.e., typically for symmetric lenses or sufficiently thin asymmetric ones with a relatively flat exit surface).

Both the KU and RS2 approximations are also surprisingly accurate for small lenses, including the microlenses, when both the lens diameter $D$ and the focal length $f$ are comparable with the radiation wavelength $\lambda(f \sim D \sim \lambda)$, though small lenses have to be symmetric for minimizing the possibility of total internal reflection effects. The KU model fails, however, for bulk asymmetric lenses with a rather convex exit surface, where the total internal reflection occurs for the waves near the lens rim.

\section{ACKNOWLEDGMENT}

The authors are grateful to J. A. Murphy for useful discussions.

\section{REFERENCES}

1. C. O'Sullivan, G. Cahill, J. A. Murphy, W. K. Gear, J. Harris, P. A. R. Ade, S. E. Church, K. L. Thompson, C. 
Pryke, J. Bock, M. Bowden, M. L. Brown, J. E. Carlstrom, P. G. Castro, T. Culverhouse, R. B. Friedman, K. M. Ganga, V. Haynes, J. R. Hinderks, J. Kovak, A. E. Lange, E. M. Leitch, O. E. Mallie, S. J. Melhuish, A. Orlando, L. Piccirillo, G. Pisano, N. Rajguru, B. A. Rusholme, R. Schwarz, A. N. Taylor, E. Y. S. Wu, and M. Zemcov, "The quasi-optical design of the QUaD telescope," Infrared Phys. Technol. 51, 277-286 (2008).

2. A. Walther, The Ray and Wave Theory of Lenses (Cambridge U. Press, 2006).

3. C. A. Balanis, Advanced Engineering Electromagnetics (Wiley, 1989).

4. P. Wenig, M. Schneider, and R. Weigel, "Performance analysis of a cylindric dielectric lens antenna for $77 \mathrm{GHz}$ Automotive Radar," in Proceedings of International Radar Symposium (IRS 2008), 21-23 May 2008, Wroclaw, Poland, A. Kawalec and P. Kaniewski, eds. (Institute of Radioelectronics, 2008), paper B1-1.

5. D. Feng, Y. Yan, G. Jin, and S. Fan, "Axial focusing characteristics of diffractive microlenses based on a rigorous electromagnetic theory," J. Opt. A, Pure Appl. Opt. 6, 1067-1071 (2004).

6. J.-S. Ye, B.-Z. Dong, B.-Y. Gu, G.-Z. Yang, and S.-T. Liu, "Analysis of a closed-boundary axilens with long focal depth and high transverse resolution based on rigorous electromagnetic theory," J. Opt. Soc. Am. A 19, 2030-2035 (2002).

7. J.-S. Ye, B.-Y. Gu, B.-Z. Dong, and S.-T. Liu, "Application of improved first Rayleigh-Sommerfeld method to analyze the performance of cylindrical microlenses with different f-numbers," J. Opt. Soc. Am. A 22, 862-869 (2005).
8. K. Duan and B. Lu, "Improved diffraction integral for studying the diffracted field of a spherical microlens," J. Opt. Soc. Am. A 22, 2677-2681 (2005).

9. M. N. O. Sadiku, Numerical Techniques in Electromagnetics (CRC, 1992)

10. C. Muller, Foundations of the Mathematical Theory of Electromagnetic Waves (Springer-Verlag, 1969).

11. D. W. Prather, M. S. Mirotznik, and J. N. Mait, "Boundary integral methods applied to the analysis of diffractive optical elements," J. Opt. Soc. Am. A 14, 34-43 (1997).

12. G. Fikioris, "A note on the method of analytical regularization," IEEE Antennas Propag. Mag. 43, 34-40 (2001).

13. S. V. Boriskina, P. Sewell, T. M. Benson, and A. I. Nosich, "Accurate simulation of two-dimensional optical microcavities with uniquely solvable boundary integral equations and trigonometric Galerkin discretization," J. Opt. Soc. Am. A 21, 393-402 (2004).

14. A. V. Boriskin, A. I. Nosich, S. V. Boriskina, T. M. Benson, P. Sewell, and A. Altintas, "Lens or resonator? Electromagnetic behavior of an extended hemielliptic lens for a submillimeter-wave receiver," Microwave Opt. Technol. Lett. 43, 515-518 (2004).

15. V. B. Yurchenko and A. Altintas, "Asymptotic wave-like modeling of dielectric lenses," in Proceedings of the 6th International Conference on Antenna Theory and Techniques (ICATT 2007), 17-21 September 2007, Sevastopol, Ukraine, Y. S. Shifrin and N. N. Kolchigin, eds. (IEEE, 2007), pp. 93-98.

16. Y. Li and E. Wolf, "Focal shifts in diffracted converging spherical waves," Opt. Commun. 39, 211-215 (1981). 